\title{
Density Measurements for Rectangular Free Jets using Background-Oriented Schlieren
}

\author{
T J Tipnis*, M V Finnis', K Knowles and D Bray§
}

\begin{abstract}
An experimental study incorporating the use of the Background-Oriented Schlieren (BOS) technique was performed to measure the density field of a rectangular supersonic jet. This technique is easier to set up than conventional schlieren since the optical alignment involving the various mirrors, lenses and knife-edge is replaced by a background pattern and a single digital camera. The acquired images which contain information of density gradients in the flow are solved as a Poisson equation and further processed using deconvolution and tomographic algorithms to generate a 3-D domain which contains information about the actual density. 2-D slices can then be extracted to quantitatively visualise the density along any required planes. The results from supersonic axisymmetric jets are used for validation of the code; these show excellent agreement with pre-validated CFD data. The results for a rectangular supersonic jet are then obtained. These show good agreement with the CFD data, in terms of shock-cell spacing and overall structure of the jet. The technique has proved useful for investigating axis-switching, a phenomenon generally associated with non-axisymmetric jets.
\end{abstract}

Keywords: Background Oriented Schlieren, BOS, density measurements, rectangular jets, axis-switching, CFD

\section{Nomenclature}

$b \quad$ Width of the rectangular nozzle exit, $m$

d Image displacement vector

$d \quad$ Image displacement, $m$

$d^{\prime} \quad$ Virtual image displacement, $m$

D Circular nozzle exit diameter, $m$

$D_{e q} \quad$ Equivalent diameter, $m ; D_{e q}=\sqrt{4 b h / \pi}$

$f \quad$ Focal length of the camera lens, $m$

$G(\lambda) \quad$ Gladstone-Dale constant, $\mathrm{m}^{3} / \mathrm{kg}$

$h \quad$ Height of the rectangular nozzle exit, $m$

$L_{s} \quad$ Shock-cell length, $m$

\footnotetext{
* Research Fellow, Department of Engineering Photonics, Cranfield University, Cranfield MK43 0AL, UK

+ Principal Research Fellow, Aeromechanical Systems Group, Cranfield University, Defence Academy of the UK, Shrivenham, SN6 8LA, UK

₹ Professor and Head of Aeromechanical Systems Group, Cranfield University, Defence Academy of the UK, Shrivenham, SN6 8LA, UK

$\S$ Senior Lecturer, Aeromechanical Systems Group, Cranfield University, Defence Academy of the UK, Shrivenham, SN6 8LA, UK
} 

M Mach number
$M_{j} \quad$ Fully-expanded Mach number at isentropic conditions for a given NPR
$n \quad$ Refractive index
$x \quad$ Horizontal direction (along axis of symmetry)
y Vertical direction
$z \quad$ Line-of-sight direction
$\beta \quad$ Angle of deflection
$\lambda \quad$ Wavelength, $m$
$\rho \quad$ Density, $\mathrm{kg} / \mathrm{m}^{3}$
$\rho_{a} \quad$ Ambient density, $\mathrm{kg} / \mathrm{m}^{3}$
$\rho_{c} \quad$ Nozzle exit centreline density, $\mathrm{kg} / \mathrm{m}^{3}$
$\begin{array}{ll}2-D & \text { Two-dimensional } \\ \text { 3-D } & \text { Three-dimensional } \\ \text { BOS } & \text { Background-oriented schlieren } \\ \text { CFD } & \text { Computational fluid dynamics } \\ \text { NPR } & \text { Nozzle pressure ratio (nozzle stagnation pressure relative to ambient static pressure) } \\ \text { PIV } & \text { Particle image velocimetry }\end{array}$

\section{Introduction}

The application of non-intrusive and quantitative techniques for the measurement of flow-fields has always been important. Optical visualisation techniques such as schlieren and shadowgraph provide qualitative information about the first and second derivatives of density, respectively. Although a schlieren or shadowgraph setup cannot be directly used as an aid to view objects based on size or distance, it assists in looking at optical inhomogeneities in a transparent medium in considerably more detail than by the naked eye. ${ }^{[1]}$ The schlieren technique was first developed by Robert Hooke using an in-line single lens arrangement with two candles. The first practical apparatus for viewing schlieren images was, however, developed by August Toeplera. It consisted of an adjustable knife-edge cutoff, a lantern as the light source and a telescope which enabled the image to be viewed directly. His test results established that temperature differences of $1^{\circ} \mathrm{C}$ were visible; this corresponded to a change in refractive index of about one part per million.

Background-oriented schlieren (BOS) derives its basic principles from the schlieren technique. It differs, however, in that it does not require the use of the extensive setup that is typical of a schlieren arrangement. It is defined as "a schlieren measurement technique based on computer

a See pp. 8, 9 in Schlieren and Shadowgraph Techniques [1], referring to the original work: Toepler, A., Beobachtungen nach einer neuen optischen Methode - Ein Beitrag zur Experimentalphysik, M. Cohen and Son, Bonn, 1964. 
evaluation of image variations due to refractive index variations in the propagation medium". [2] The variation in density of the transparent medium affects the refractive index; this is the principle on which the BOS technique is based. The relation between the density and the refractive index, first proposed by Gladstone and Dale ${ }^{[3]}$ can be written as:

$$
\frac{n-1}{\rho}=G(\lambda)
$$

This is referred to as the Gladstone-Dale equation, where $n$ is the refractive index of the fluid, $\rho$ is the density of the medium, and $G(\lambda)$ is the Gladstone-Dale constant. The Gladstone-Dale constant is dependent on certain characteristics of the medium and weakly on the frequency of the light used. The reader is directed to Merzkirch [4] for more detail.

The distorted image of a particular background is compared numerically with an undistorted one to determine the gradients of density. The BOS technique offers the possibility of determining the density field by integration of the measured gradients. A specific illuminated background pattern and a digital camera are the only instruments required for this arrangement. Consequently, it is easier to set up than conventional schlieren. Different background patterns provide different results for correlation, depending on the contrast of the captured image and the flow direction. The best results are, thus, usually generated using a random dot background since it has high contrast and unique features which are ideal for image correlation algorithms to determine pixel shift. [5]

The BOS method has been successfully employed as a quantitative measurement technique for different applications, ranging from under-expanded axisymmetric jets [6], multi-jet flows [6] [7], hypersonic shock waves [8], supersonic separated flow [9], and shock waves of a rifle discharge and explosions [5], to name a few. The current study aims to make use of the BOS technique to look at the flow in an under-expanded rectangular jet and understand its development downstream of the nozzle exit.

\section{Technique}

Typically, as a first step, an image is taken of the random dot pattern with the medium (air) at rest, i.e. no-flow condition. The second image is then taken with the nozzle running at the required nozzle pressure ratio (NPR). This image contains the necessary density gradients that distort the background image. A cross-correlation algorithm is employed to generate the displacements of the dots between the two images in the $x$ and $y$ directions. In the current setup $x$ is along the centreline of the jet downstream of the exit, with the origin at the centre of the exit. The vertical axis is denoted by $y$, while $z$ is the line-of-sight direction. It can be shown [1] that refraction of light passing through a medium is proportional to the gradient of the refractive index. Thus, the deflection of a single beam contains information about the spatial gradient of the refractive index integrated along the axial path. ${ }^{[10]}$ Thus, the image deflection $\beta$ is given by:

$$
\beta=\frac{1}{n_{0}} \int_{a-\Delta a}^{a+\Delta a} \frac{\delta n}{\delta y} d z \propto \frac{\delta n}{\delta y}
$$

(where $n_{0}$ is the refractive index of undisturbed air). This is illustrated in Fig. 1 which shows the typical BOS arrangement. The background pattern is positioned at $B$, the density field to be measured is positioned at $S$, the camera lens is at $L$ and the image plane is positioned at $I$. The solid line indicates one undistorted image ray $R 1$ while one distorted image ray, $R 2$, is shown by a dashed line. 


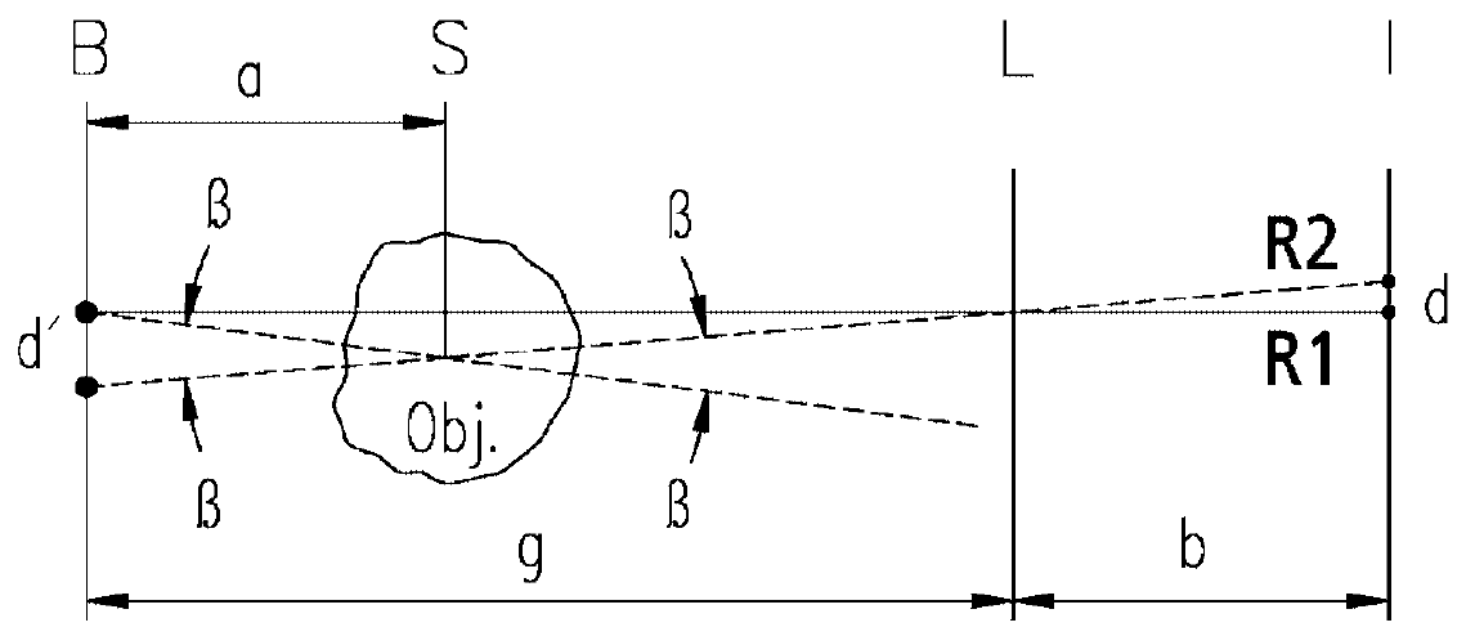

Figure 1: A representative diagram for typical BOS setup [2]

From the figure, using simple geometrical reasoning, we can see that the virtual displacement $d^{\prime}$ is related to the image displacement $d$ by the distance of the background from the lens $g$ and the distance of the image plane from the lens $b$. For large $g$, this may be replaced by the focal length of the camera lens $f$. Thus we have,

$$
\frac{d^{\prime}}{g}=\frac{d}{b}=\frac{d}{f}
$$

Also, if the deflection angle $\beta$ is considered very small, it may be approximated as $d^{\prime} / a$, where $a$ is the distance of the optical disturbance from the background. Using this approximation, Eqn. 3, and the proportionality relation of Eqn. 2, the sensitivity for a given setup is stated as:

$$
\left(\frac{d}{\delta n / \delta y}\right) \propto\left(\frac{a f}{g}\right)
$$

Thus, the shift of the background and, hence, the sensitivity increases with increasing distance of the density gradient from the background. For a constant image field, this implies that the increased distance between the background and lens, $g$, may then be compensated by increasing the focal length of the camera lens, $f$.

Extending to two dimensions and using Eqn. 1, we have:

$$
\nabla n \propto \nabla \rho \propto \mathbf{d}
$$

The obtained displacements are, therefore, proportional to the density gradients at each point in the field. Applying the div operator, we get:

$$
\nabla^{2} n \propto \nabla . \mathbf{d}
$$

Rewriting this, we get the Poisson Equation of the form:

$$
\frac{\partial^{2}}{\partial x^{2}} \rho(x, y)+\frac{\partial^{2}}{\partial y^{2}} \rho(x, y)=S(x, y)
$$

where $S(x, y)$, the source term, is obtained from the observed displacements and the constants of proportionality derived from the experimental geometry and Eqn. 1. In evaluating the source term at each mesh point, the displacements are obtained through correlation using standard PIV 
techniques ${ }^{[11]}$ and differentiated numerically. Neumann boundary conditions, i.e. $\nabla \rho=0$, are applied during the initiation of the solving process. The solution of Eqn. 7 yields the line-of-sight integrated density field, i.e. the three-dimensional density field as projected when viewing from one direction. The projected density fields, obtained from observing the phase object along different directions, can then be simultaneously deconvoluted using optical tomographic techniques (e.g. filtered backprojection technique). This enables the density distribution to be obtained for any given plane. More details on the filtered backprojection technique applied to BOS may be obtained from Venkatakrishnan and Meier. [10] In particular, for details about various tomographic techniques available, the reader is referred to Kak and Slaney. ${ }^{[12]}$

\section{Setup}

The experiments were carried out using Cranfield University's jet rig facility at the Defence Academy of the UK in Shrivenham. Compressed air was supplied using a CompAir L110-10A screw-type compressor. The pressure in the system was limited to 7 bar(g); the maximum operable pressure for continuous running of the jet rig was $3.5 \operatorname{bar}(\mathrm{g})$. A circular settling chamber of internal diameter $203 \mathrm{~mm}$ was used to straighten the flow and reduce swirl, at the same time filtering the air so that any particulates would not damage the nozzle. A spline section reduced the internal diameter of the settling chamber to $65 \mathrm{~mm}$. The rectangular nozzle used for the experiments had a circular inlet of $35 \mathrm{~mm}$ diameter and an exit of aspect ratio 4 and area of $400 \mathrm{~mm}^{2}$. It was affixed using an aluminium adapter to reduce the diameter from $65 \mathrm{~mm}$ to $35 \mathrm{~mm}$. For the reconstruction of the 3-D density field, projections were required to be taken from different directions and then deconvoluted simultaneously. Since the entire setup involving the camera and the background could not be rotated easily around the nozzle, the rectangular nozzle was instead rotated using a dual-flange arrangement (Fig. 2). This enabled taking measurements from $0^{\circ}$ through to $90^{\circ}$ azimuth. Using symmetry of the nozzle about its axes, the measurements from $90^{\circ}$ to $180^{\circ}$ azimuth were replicated from the measurements obtained in the first quadrant.

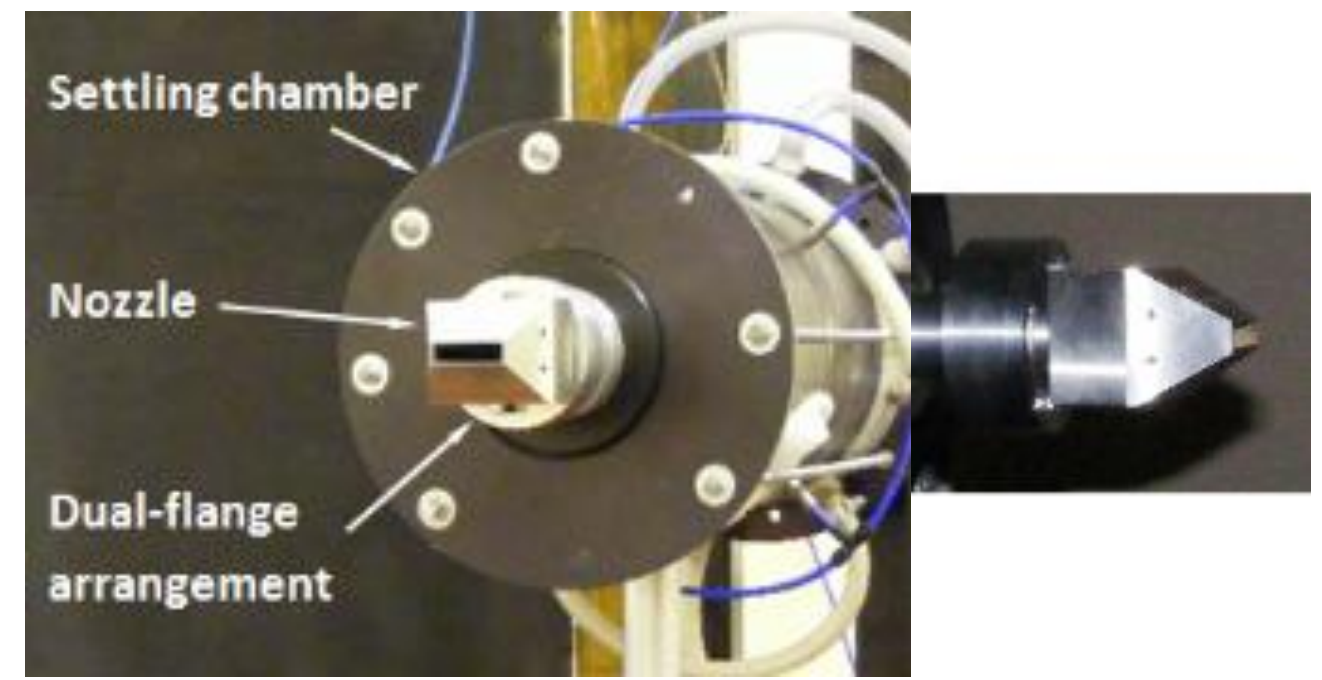

Figure 2: The dual-flange arrangement used for rotating the rectangular nozzle through $90^{\circ}$ (left), closer view (right)

A schematic representation of the experimental setup is given in Fig. 3. The background was created using a random dot pattern-generator program, appropriately modified to generate the pattern for A1-sized paperb. This was laminated and mounted on a frame made of aluminium

b The authors would like to acknowledge Dr Frédéric Moisy, Assistant Professor, Université Paris-Sud, the originator of the Matlab program for creating a random dot background pattern for A4-sized paper. The program is available to download freely at http://www.fast.u-psud.fr/ moisy/ml/misc/makebospattern.m. The program was modified by the authors to adapt it for the current study. 
extrusions to ensure rigidity. A halogen lamp placed approximately $1.5 \mathrm{~m}$ away was used to illuminate the pattern. This approximated parallel beam conditions and, thus, eliminated the need to re-sort the fan-beam projections into parallel beam projections. [10] [12]

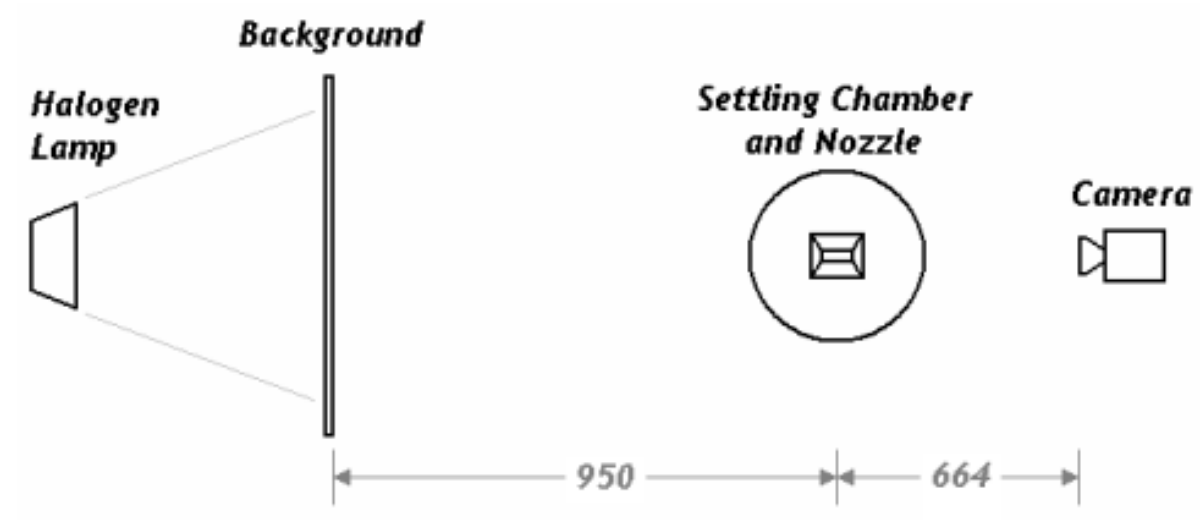

Figure 3: Schematic representation of the experimental setup; dimensions are given in $\mathbf{m m}$

Initial experiments were conducted using a compact camera having a small sensor; however, it was soon realised that results would be improved using a larger sensor. An image sensor with a higher pixel resolution is able to capture more detail in the image. This helps in improving the accuracy of the density gradients calculated from the image cross-correlation. Coupled with a larger sensor, which corresponds to better signal levels and less noise, the image quality improves significantly. These factors make using a digital single-lens reflex camera suitable for BOS studies. The images were captured in RAW format by a Canon EOS350D digital camera (8 Megapixel sensor) with an $18-55 \mathrm{~mm}$ lens set at a focal length ratio $\left(f_{\#}\right)$ of 3.5 , focal length $(f)$ of approximately $41 \mathrm{~mm}$ and an exposure time of $1 / 30 \mathrm{~s}$, using a remote trigger. The remote trigger ensured that the camera, mounted on a tripod, would not be subject to any physical displacement due to manually operating the shutter. The aperture setting on the camera allowed maximum light to be captured during the imaging process. Although this setting would correspond to a lower depth of field, it actually allows the focus to be set more precisely on the background, which is a requirement for the BOS technique.

\section{Methodology}

\subsection{Image Acquisition}

The density data were calculated based on the acquisition of the image pair with no-flow and flow conditions. The image capture in terms of overall experiment setup was the same for both axisymmetric and rectangular nozzles. There were, however, small differences in the methods due to differences in the flow-fields observed.

\subsubsection{Axisymmetric Nozzle}

For an axisymmetric nozzle, the density projections along any direction normal to the streamwise axis are identical. Therefore, only one set of data is required for calculation of the gradients and the integrated density field. With the setup in place, an image was acquired for the no-flow condition (Fig. 4a). This served as the base image for cross-correlation. Another image was acquired with the nozzle running at the required NPR (Fig. 4b). This process was repeated four times to give five image pairs. These image pairs were then averaged and used sequentially for 180 projections corresponding to an azimuthal angular increment of $1^{\circ}$ from $0^{\circ}$ to $179^{\circ}$. The axisymmetric nozzle was run at NPR $=3.00$ for this particular study. 


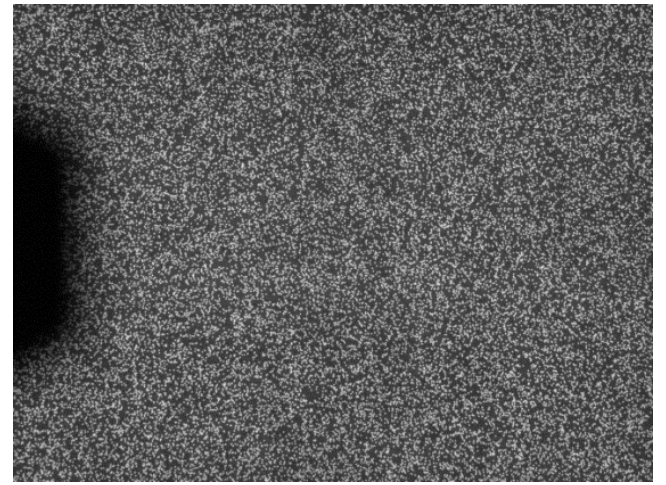

(a) Without flow

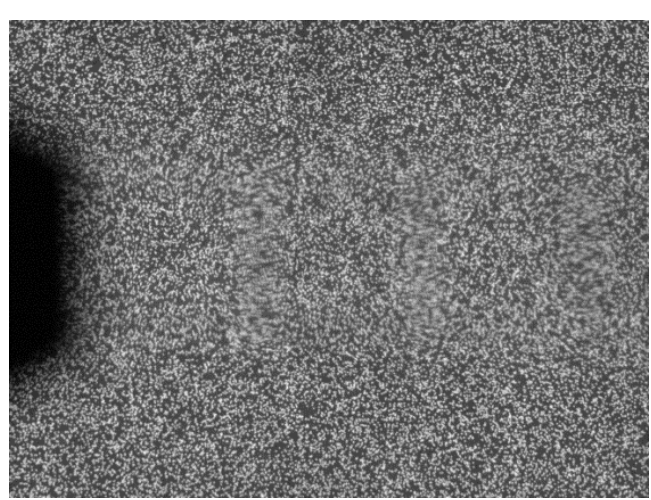

(b) With flow

Figure 4: The background dot pattern image pair used for creating the vector field

\subsubsection{Rectangular Nozzle}

The axisymmetric flow-field presents simplicity, in that a single data set is sufficient to reproduce the entire 3-D flow field. Such is not the case with a rectangular nozzle flow-field. There are two ways of accomplishing the task of acquiring projections of a rectangular flowfield: rotating the measurement setup about the centreline of the jet, or rotating the nozzle about its centreline axis. The former, although ideal in terms of ensuring that the flow remains undisturbed, is impractical since it involves re-orientation of the camera and the background and is physically difficult to set up, particularly with a fairly large-scale rig. The second method requires the nozzle to be rotated about its centreline, keeping the rest of the setup intact. This is more feasible and easier to achieve with appropriate modifications.

The dual-flange arrangement was used to rotate the nozzle from $0^{\circ}$ to $90^{\circ}$ so that projections could be taken without disturbing the background and the camera positions. The symmetry of the nozzle about its major and minor axes allowed the assumption that the projections of the density for $90^{\circ}$ to $180^{\circ}$ would be the same as those for $90^{\circ}$ to $0^{\circ}$. The projections were taken in steps of $7.5^{\circ}$ from $0^{\circ}$ (minor axis) through to $90^{\circ}$ (major axis), resulting in 13 projections. The data for projections from $7.5^{\circ}$ to $82.5^{\circ}$ were assumed to be the same for projections between $172.5^{\circ}$ to $97.5^{\circ}$, based on symmetry about its major axis; the total number of projections being 24. Each projection resulted in a different line-of-sight density field which was then analysed and processed to produce the 3-D density field. The rectangular nozzle was run at NPR $=2.50$ for this study.

\subsection{Image Processing}

Clearly, the higher the number of projections, the better is the resolution of the deconvoluted density field. The image pairs (no-flow and flow) were analysed and processed initially using a multi-step vector-analysis code (written in FORTRAN). This code computed the density gradients using a cross-correlation algorithm ${ }^{[11]}$ and stored the data in the form of an array. The density was then obtained by numerical integration of the Poisson Equation (Eqn. 7); the recorded density of the ambient medium was used to initialise the solution. This yielded the integrated line-of-sight density field (Fig. 5). The data were then processed by a separate code for deconvolution and tomographic reconstruction of the projected density data (written in Matlab). Using the filtered backprojection method (FBP), the density of the flow-field was calculated. This code incorporated the use of the built-in 'Inverse Radon Transform' function. [12] The necessary inputs for the variables in the function were appropriately inserted for the current work. The Shepp-Logan filter ${ }^{[13]}$ was used for FBP in this study. This filter combines a sinc function (the continuous inverse Fourier transform of a rectangular pulse of unit height and $2 \pi$ width) with the ramp filter. Consequently, it is much less sensitive to noise. ${ }^{[10]}$ The entire 
density field was then normalised using the centreline density at the nozzle exit $\left(\rho_{c}\right)$ and the resultant non-dimensionalised density stored in the form of a three-dimensional array. This enabled easy extraction of data along any required 2-D plane and could be accomplished by using any appropriate flow visualisation software.

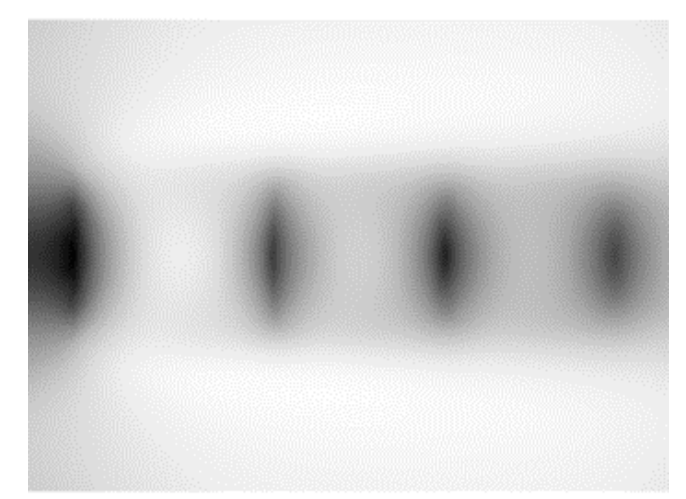

Figure 5: The projected density field, as computed from the Poisson equation

\subsection{CFD Simulations}

The CFD studies were initially conducted for a jet running at NPR $=2.50$. The RNG $\mathrm{k}-\varepsilon$ turbulence model was used since it has been found to be the most suitable amongst the k- $\varepsilon$ models for modelling under-expanded turbulent jets. [14] The mesh was tested for grid independence and suitably adapted such that the wall $y^{+}$values at the inner wall of the nozzle were approximately between 1 and 5 . Second-order discretization schemes were employed for the solution of the pressure and turbulence quantities. The pressure outlet downstream of the nozzle was specified at 1 bar. The CFD results were validated by comparing the data from previously published work [15] for axisymmetric under-expanded jets at similar NPR values. The non-dimensionalised results for velocity and pressure profiles along the centreline showed good agreement for the given NPR (Fig. 6).

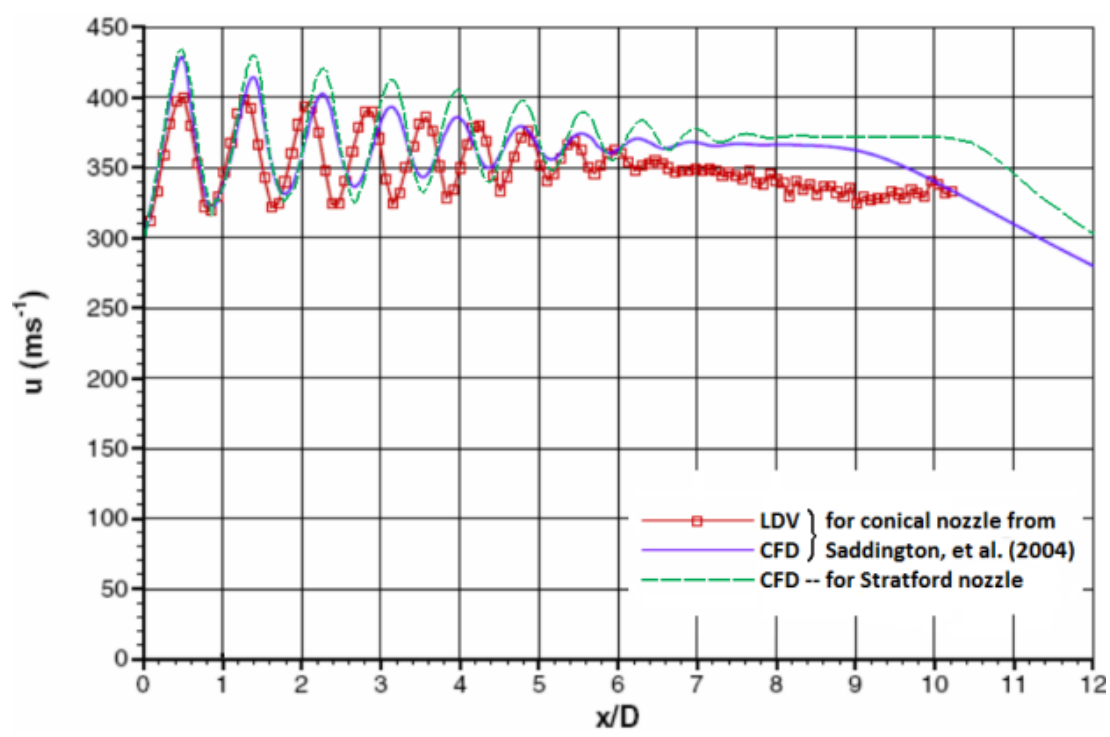

Figure 6: Comparison of jet centreline axis velocity profiles with results from Saddington, et al. (2004) [15] for the axisymmetric nozzle running at $\mathrm{NPR}=\mathbf{2 . 5 0}$ 
The Prandtl-Pack formula [16] for theoretical calculation of the under-expanded shock-cell spacing of an axisymmetric jet is given as:

$$
L_{s}=1.306\left(M_{j}^{2}-1\right)^{1 / 2} D
$$

For an under-expanded jet at NPR $=2.50$, the corresponding fully-expanded Mach number at isentropic flow conditions $\left(M_{j}\right)$ is 1.223 . The shock-cell spacing thus calculated is $0.92 \mathrm{D}$, which agrees very well with the CFD calculation of $0.89 \mathrm{D}$.

Following the validation of the CFD at NPR $=2.50$, another simulation was then set up with similar conditions for a nozzle flow at NPR $=3.00\left(M_{j}=1.358\right)$. The shock-cell structure was compared with the schlieren image acquired at the same NPR (Fig. 7). The two show a very good agreement with the shock-cell spacing at approximately 1.16 D for the CFD and 1.19 D for the schlieren. The theoretically calculated shock-cell spacing at this NPR, from Eqn. 8, is $1.20 \mathrm{D}$, matching very well with the experimental and numerical results.

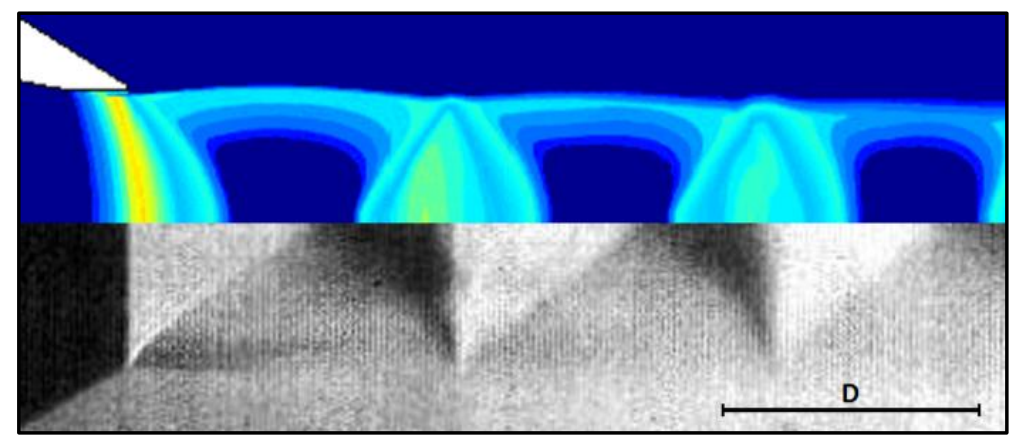

Figure 7: Shock-cell spacing comparison for the CFD simulation (top) with a conventional schlieren image (bottom) for the axisymmetric nozzle running at NPR $=3.00$

The density data from the CFD simulation at NPR $=3.00$ were then used for a quantitative comparison with the BOS results and their validation. This is discussed in the next section.

\section{Results and Discussion}

The validation of the process and the codes was carried out using data obtained from previous conventional schlieren studies based on a circular, truncated (convergent) "Stratford" nozzle [17] with exit diameter of $25 \mathrm{~mm}$, operating at NPR $=3.00$. For a quantitative assessment of the results (as mentioned in Sec. 4.3), a comparison is also given with a CFD study for the same nozzle.

Following the acceptance of the process and the codes for quantitatively assessing the density field, it was then implemented for studying the rectangular jet running at NPR $=2.50$. These results were then compared to conventional schlieren results and CFD studies for the same NPR.

\subsection{Axisymmetric Nozzle}

Fig. 8 shows the comparison between the density contours calculated using BOS and a conventional schlieren image. The two results show good agreement in terms of the shock-cell spacing for the first shock-cell (approximately 1.19 D for the schlieren and 1.17 D for BOS). The quantitative values of the density field are then compared with the data available from the CFD simulation (Fig. 9). While the overall figures match quite favourably, there is, however, a slight distortion in the values calculated by BOS. This may be due to the susceptibility of the SheppLogan filter to a small amount of blurring. [13] 


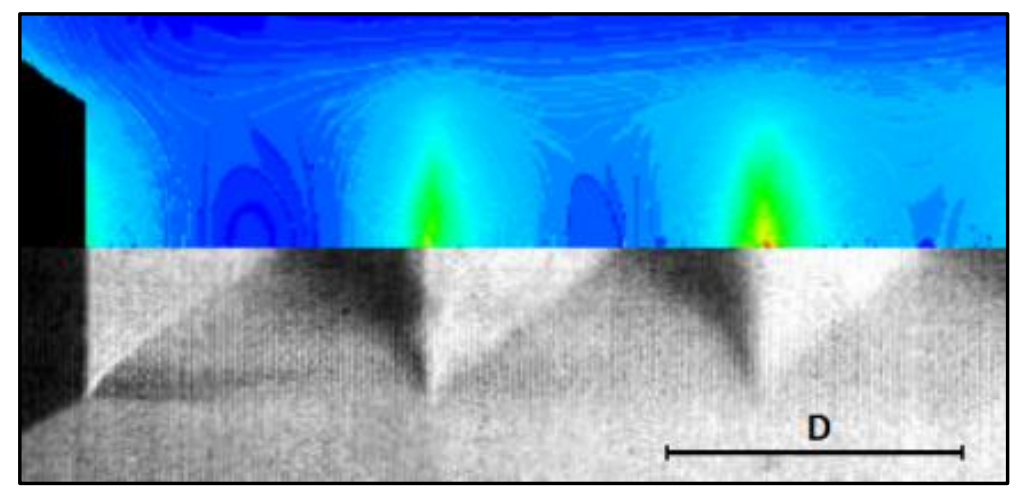

Figure 8: Shock-cell spacing comparison for the axisymmetric nozzle running at NPR = 3.00; BOS result (top) with a conventional schlieren image (bottom)

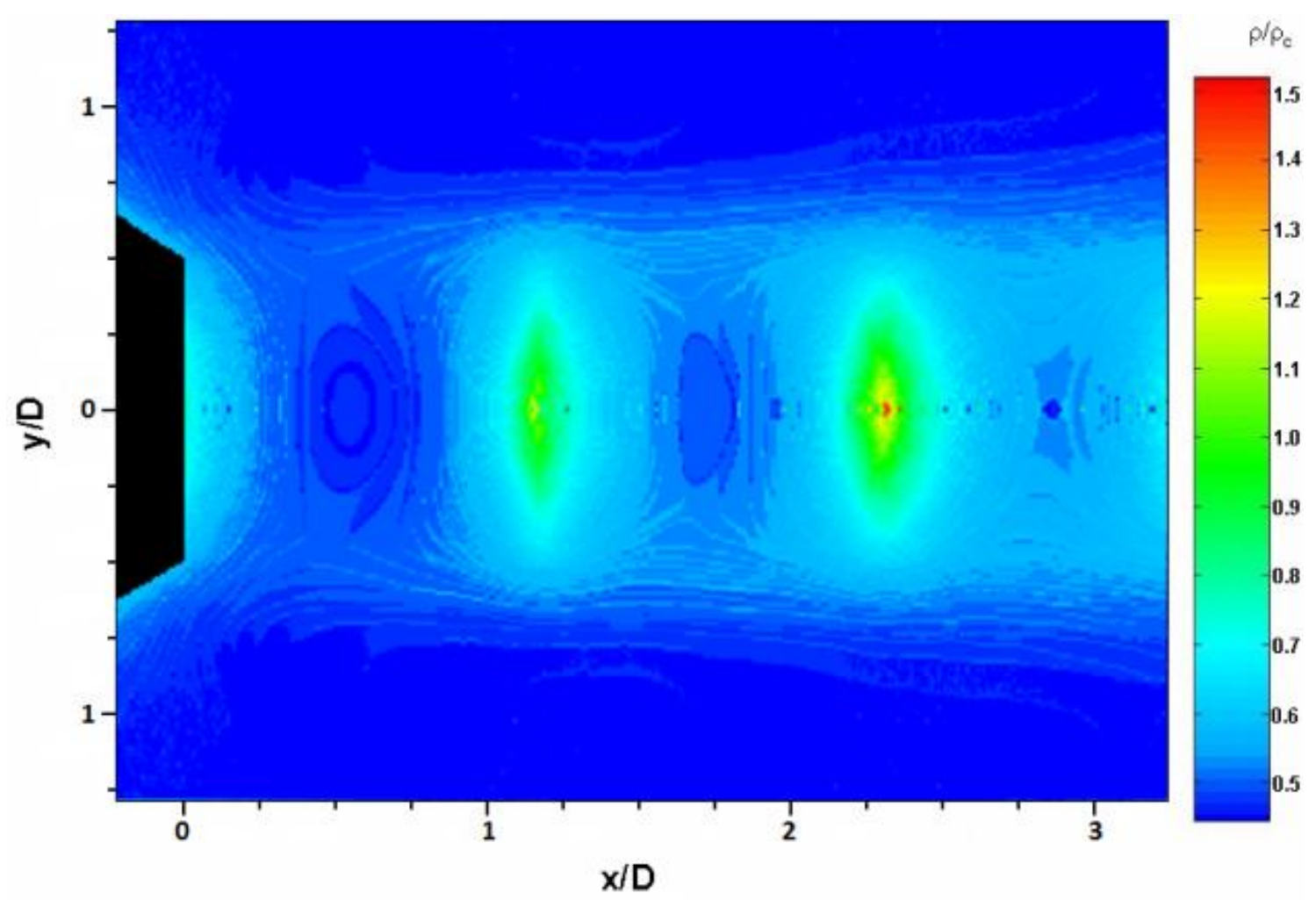

(a) Background-oriented schlieren

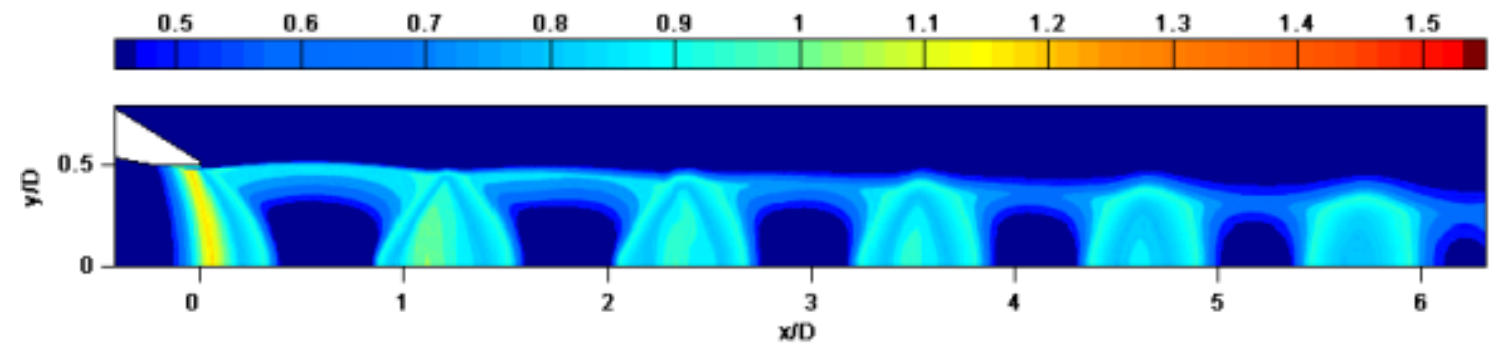

(b) CFD

Figure 9: Comparison of normalised density contours for the axisymmetric nozzle running at NPR = 3.00; $\rho_{c}$ is the nozzle exit centreline density

These results reflect the effectiveness of the application of BOS for the quantitative study of a circular nozzle density field. The process of image acquisition and post-processing using the 
multi-step vector-analysis, and the deconvolution and tomographic reconstruction codes was effectively the same for the rectangular nozzle. The validation of the BOS technique for the axisymmetric nozzle case thus implies that the post-processing is acceptable for the rectangular nozzle BOS setup.

\subsection{Rectangular Nozzle}

While the axisymmetric nozzle results were generated using 180 projections, the rectangular nozzle results, initially, were generated using 24 projections. This resulted in a significantly lower resolution for the density field calculated using BOS.

The shock-cell structure shows good agreement for the rectangular nozzle flow (Fig. 10). The values of density calculated using BOS are slightly higher compared to the CFD results (Fig. 11); the existence of the very high density regions is most likely due to distortion and blurring, and an insufficient number of projections.

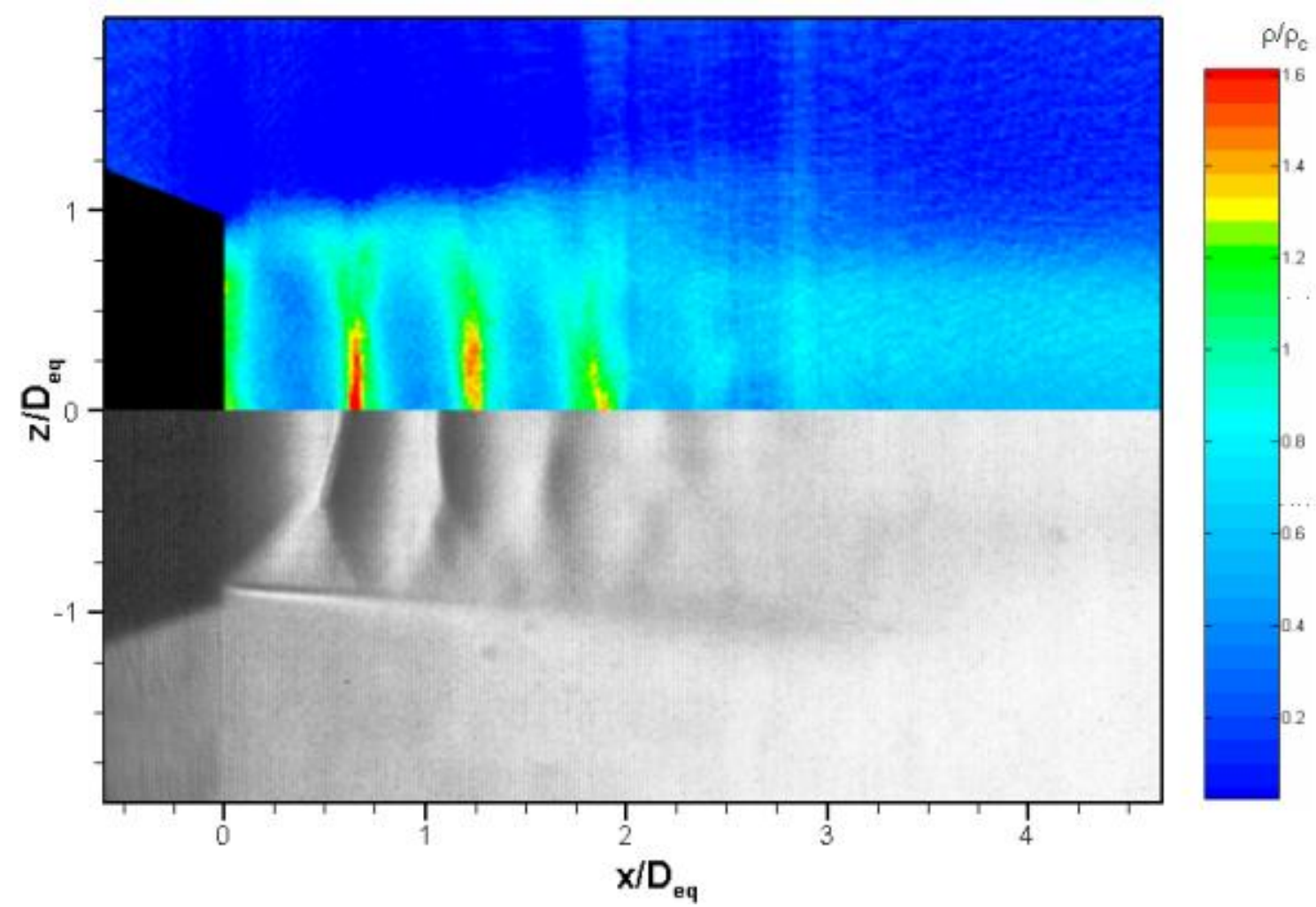

Figure 10: Shock-cell spacing comparison along the major axis plane for the rectangular nozzle running at NPR = 2.50; BOS result (top), conventional schlieren iamge (bottom)

To improve the resolution of the 3-D density field, another set of images was acquired for the rectangular nozzle at the same NPR $=2.50$. In this case, the projections were taken in steps of $5^{\circ}$. Based on the procedure detailed above, this resulted in a total of 36 projections, which were further processed using the codes to generate the required density field. The data were used to visualise the jet flow as it developed downstream of the exit. Fig. 12 shows the development of the jet by tracking the shape of the jet spreading, indicated by an iso-line of normalised density $\left(\rho / \rho_{a}\right)$, equal to 1 . It can be seen that after exiting the nozzle, the jet spreads faster along the minor axis than the major axis. This leads to the jet cross-section appearing to have rotated about the centreline through approximately $90^{\circ}$ at some distance downstream of the exit; this phenomenon is known as 'axis-switching'. [18] Clearly, the supersonic rectangular jet in this case exhibits such a behaviour. The cross-over between the axes appears to have occurred around 5 $D_{\text {eq }}$ downstream of the exit. 


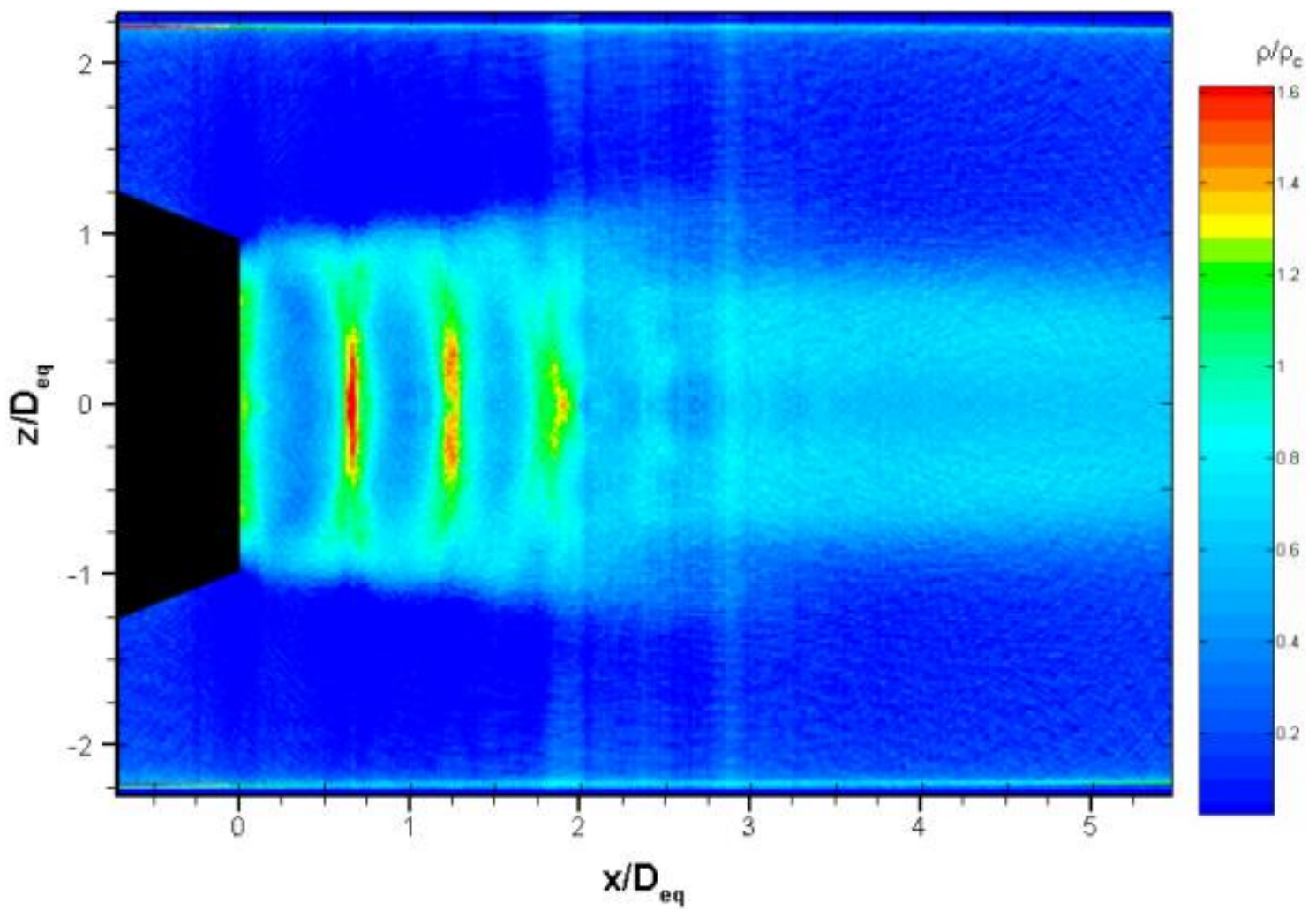

(a) Background-oriented schlieren

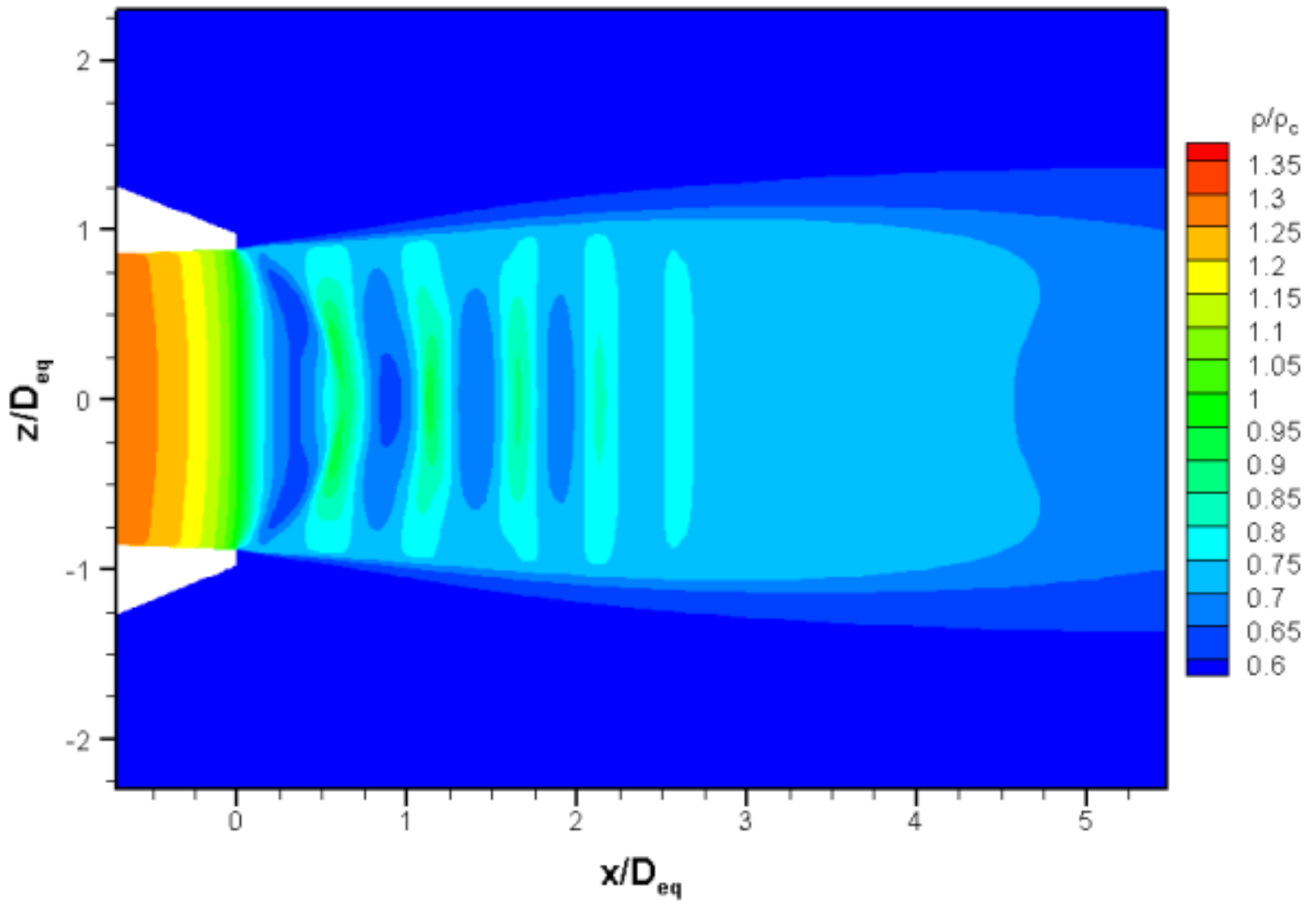

(b) CFD

Figure 11: Comparison of normalised density contours along the major axis plane for the rectangular nozzle running at NPR $=2.50 ; \rho_{c}$ is the centreline density at the nozzle exit 


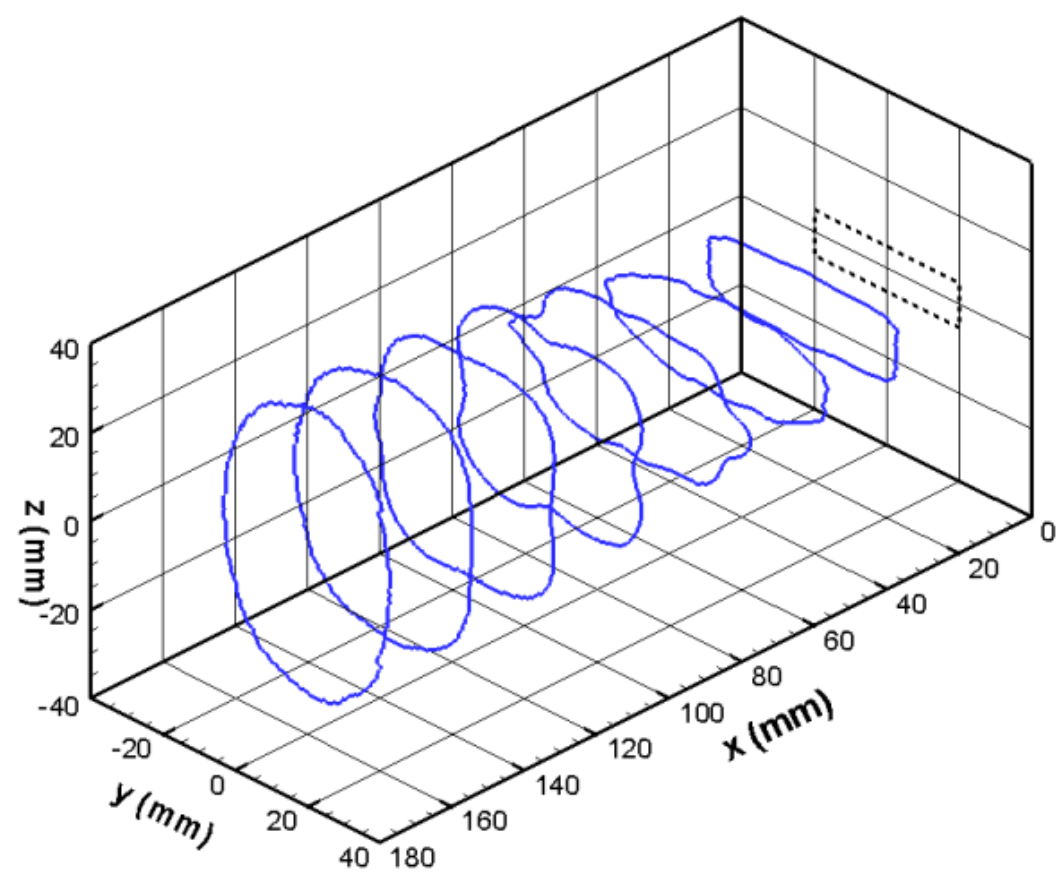

Figure 12: Jet development indicating 'axis-switching' for the supersonic rectangular jet (NPR = 2.50); the blue (solid) iso-lines indicate normalised density contours $\left(\rho / \rho_{a}\right)$ equal to 1 , given for equispaced locations from 1 to $7 D_{\text {eq }}$ downstream of the nozzle exit; the exit is indicated by the dashed outline

\section{Conclusions}

The background-oriented schlieren method is easy to set up as compared to many other quantitative measurement techniques. It is a completely non-intrusive method of measuring a flow-field property and, with proper calibration, is ideal for measurement of axisymmetric flow experiments. It is, however, limited in its application. For measurement of non-axisymmetric flow, there has to be an appropriate arrangement to move the background and the camera setup without disturbing their positions with respect to each other. Alternatively, the same result might be achieved if the flow-field to be measured can be rotated about a fixed axis without affecting the flow structures developing, as was done for the current experiment. It also requires a sufficiently large space unobstructed by any other equipment so that the images captured exhibit only the flow and its development.

In spite of these inherent drawbacks, it still shows promise for non-intrusive measurement. A number of lessons have been learnt from the current study.

- The resolution of the digital camera affects the level of detail in the image; greater sensor size results in lower noise levels and thus better image quality.

- Since a uniformly back-lit pattern is the most ideal for the background, improvements could be made by using a more diffused source of lighting with higher illumination. This would also ensure that the projections are emitted in a truer parallel-beam condition.

- The BOS method shows potential to be applied for the quantitative study of supersonic non-axisymmetric jet flow-fields. If employed correctly, it can be a very useful tool for visualising flow features such as axis-switching, as demonstrated in this study.

\section{Acknowledgements}

The authors would like to thank Dr Graham Stabler (formerly Lecturer, Cranfield University) and Dr N. J. Lawson (Reader and Pilot, Cranfield University) for their insight and comments regarding the experimental setup and applications. 


\section{References}

[1] G. S. Settles, Schlieren and Shadowgraph Techniques, $1^{\text {st }}$ ed., Springer-Verlag, 2001.

[2] G. E. A. Meier, "Computerized background-oriented schlieren," Experiments in Fluids, vol. 33, no. 1, p. 181-187, 2002.

[3] J. H. Gladstone and T. P. Dale, "Researches on Refraction, Dispersion and Sensitiveness of Liquids," Philosophical Transactions of the Royal Society of London, vol. 153, p. 317-343, 1863.

[4] W. Merzkirch, Flow Visualization, $2^{\text {nd }}$ ed., Academic Press, 1987.

[5] M. J. Hargather and G. S. Settles, "Natural-background-oriented schlieren imaging," Experiments in Fluids, vol. 48, no. 1, p. 59-68, 2010.

[6] L. Venkatakrishnan, "Density measurement in an axisymmetric underexpanded jet using Background-oriented Schlieren technique," AIAA Journal, vol. 43, no. 7, p. 1574-1579, 2005.

[7] E. Goldhahn and J. Seume, "The background oriented schlieren technique: sensitivity, accuracy, resolution and application to a three-dimensional density field," Experiments in Fluids, vol. 43, no. 2-3, p. 241-249, 2007.

[8] D. Ramanah, S. Raghunath, D. J. Mee, T. Rösgen and P. A. Jacobs, "Background oriented schlieren for flow visualisation in hypersonic impulse facilities," Shock Waves, vol. 17, no. 1-2, p. 65-70, 2007.

[9] L. Venkatakrishnan and P. Suriyanarayanan, "Density field of supersonic separated flow past an afterbody nozzle using tomographic reconstruction of BOS data," Experiments in Fluids, vol. 47, no. 3, p. 463-473, 2009.

[10] L. Venkatakrishnan and G. E. A. Meier, "Density measurements using the Background Oriented Schlieren technique," Experiments in Fluids, vol. 37, no. 2, p. 237-247, 2004.

[11] M. Raffel, C. Willert, S. Wereley and J. Kompenhans, Particle Image Velocimetry - A Practical Guide, 2nd ed., Springer-Verlag, 2007.

[12] A. C. Kak and M. Slaney, Principles of Computerized Tomographic Imaging, New York: IEEE Press, 1988.

[13] L. A. Shepp and B. F. Logan, "The Fourier reconstruction of a head section," IEEE Transactions on Nuclear Science, vol. 21, p. 21-43, 1974.

[14] K. Knowles and A. J. Saddington, "Modelling and experiments on underexpanded turbulent jet mixing," in Engineering Turbulence Modelling and Experiments 5, Elsevier, Oxford, p. 789-798, 2002.

[15] A. J. Saddington, N. J. Lawson and K. Knowles, "An experimental and numerical investigation of under-expanded turbulent jets," The Aeronautical Journal, vol. 108, no. 1081, p. 145-152, 2004.

[16] D. Pack, "A note on Prandtl's formula for the wavelength of a supersonic gas jet," The Quarterly Journal of Mechanics and Applied Mathematics, vol. 3, no. 2, p. 173-181, 1950.

[17] B. S. Stratford, "The calculation of the discharge coefficient of profiled choked nozzles and the optimum profile for absolute air flow measurement," Journal of the Royal Aeronautical Society, vol. 68, p. 237-245, 1964.

[18] F. Grinstein, "Entrainment, axis switching, and aspect-ratio effects in rectangular free jets," in $4^{\text {th }}$ Shear Flow Control Conference, Snowmass Village, CO, USA, AIAA-1997-1875, 1997. 